\title{
Assessment of the Quality of Care of Patients with Diabetic Emergencies Admitted in the Philippine General Hospital
}

\author{
Sahra May O. Paragas and Laura Trajano-Acampado \\ Division of Endocrinology, Diabetes and Metabolism, Department of Medicine, College of Medicine and Philippine General Hospital, \\ University of the Philippines Manila
}

\begin{abstract}
Objective. This is a health systems research conducted with the goal of evaluating the quality of care (QoC) in diabetic emergencies, specifically Diabetic Ketoacidosis (DKA) and Hyperglycemic hyperosmolar state (HHS), at the Philippine General Hospital (PGH) in terms of structures, processes and outcomes, and determining facilitators and barriers to effective delivery of care from the healthcare providers' point of view.

Methods. The first phase of this study is a retrospective chart review involving an audit of the quality of services rendered to patients diagnosed to have DKA/HHS at the PGH. The second phase is a series of focus group discussions (FGDs) among physicians and nurses involved in the care of DKA/HHS patients. Facilitators and barriers to delivery of care were identified in these FGDs, as well as recommendations on how to improve delivery of care.
\end{abstract}

Results. The recognition of DKA/HHS as a possible diagnosis at first encounter was observed in only $67 \%$ of cases. Timely initiation of hydration was met in $40 \%$ of cases and only $10 \%$ of the patients underwent adequate laboratory monitoring. Correction of at least half of the estimated water deficit in the first 24 hours of admission was achieved in $84 \%$ of the cases. Despite this, mortality rate was still high at $23 \%$. Among those who died, thirtyseven percent (37\%), seventy-five percent (75\%) and over thirty percent (31\%) had delayed initiation of hydration, at least one episode of hypokalemia or hypoglycemia, respectively. Barriers to good quality of care for DKA/HHS were mostly attributed to delays. These delays were due to lack of resources, limited bed-capacity and challenges experienced in the handling of specimen from the ER clerk to the laboratory and release of results.

Conclusion. Failure to follow guidelines and delays in the delivery of care are possible reasons for the high mortality rates noted and could be a reflection of poor quality of care among DM emergency patients in PGH. Proper documentation in the medical charts is also important. Clinical pathways and DM emergency kits are a few of the suggested approach to address the barriers to good quality care.

Key Words: Diabetic ketoacidosis (DKA), hyperglycemic hyperosmolar state (HHS), quality of care (QoC)

\section{INTRODUCTION}

Corresponding author: Sahra May O. Paragas, MD Division of Endocrinology, Diabetes and Metabolism Department of Medicine

Philippine General Hospital

University of the Philippines Manila

Taft Avenue, Manila 1000, Philippines

Email: sahraparagas@gmail.com
Diabetic emergencies, specifically diabetic ketoacidosis (DKA) and hyperglycemic hyperosmolar state (HHS), are acute complications occurring in both type 1 and type 2 diabetes mellitus. ${ }^{1,2}$ DKA is defined as blood glucose $>250$ $\mathrm{mg} / \mathrm{dL}$, arterial $\mathrm{pH} \leq 7.30$, bicarbonate level $\leq 18 \mathrm{mEq} / \mathrm{L}$, and adjusted for albumin anion gap $>10-12.3$, where a positive serum and urine ketone result may further support the diagnosis. ${ }^{3}$ HHS on the other hand is characterized by plasma glucose $>600 \mathrm{mg} / \mathrm{dl}$, arterial $\mathrm{pH}>7.3$, serum bicarbonate $>18$, effective serum osmolarity $>320 \mathrm{mOsm} / \mathrm{kg}$ and more pronounced mental status change. ${ }^{4}$ Available data for DKA show mortality rates at $<1 \%-1.8 \%$ in the United 
Kingdom, $<1 \%$ in the United States, $0.65 \%-3.3 \%$ in Canada, and up to as high as $30 \%$ in countries with "less privileged health systems." ${ }^{-8}$ Even though its admission rates are lower than DKA, HHS was noted to have higher mortality rates. ${ }^{1}$

In a tertiary government training hospital in the Philippines, a study showed a 5\% incidence rate for DKA and mortality rate as high as $11 \%$, where infection was identified as the most common precipitating factor accounting for $38 \%$ of cases, while non-adherence to diabetes treatment, either oral hypoglycemic agents or insulin, accounted for 15\%. ${ }^{9}$ Key to successful resolution of these emergencies include early recognition and diagnosis, comprehensive clinical and biochemical evaluations, and effective management. ${ }^{1}$ Essential to the latter are adequate fluid resuscitation, gradual reduction of blood glucose and plasma osmolarity, correction of electrolyte imbalances, prompt identification and correction of precipitating factors, and close monitoring of the patient with use of laboratory procedures to assess resolution. ${ }^{1}$

Guidelines have been formulated to help clinicians manage diabetic emergencies. These provide vital information for healthcare providers and serve as guide in tailor-fitting an approach to adequately manage the cases while avoiding treatment induced complications, including hypokalemia and hypoglycemia. ${ }^{1}$ Gosmanov et al, provided a checklist for DKA management milestones that need to be achieved within a prescribed time such that at the $12^{\text {th }}$ to $24^{\text {th }}$ hour of admission, a patient should be adequately hydrated, blood sugar controlled, electrolyte imbalance corrected, precipitating factors identified and addressed, and referred to a diabetes educator. ${ }^{3}$ However, in a study by Singh et al, the recommended standard of care in the management of DKA was met only in $22.5 \%$ of cases. ${ }^{5}$ Timely initiation of IV fluid within 30 minutes of admission was observed in $30 \%$ of cases and initiation of IV insulin within 60 minutes was 31\%. Gatbonton et al in 1998 found, in a government hospital in the Philippines, that the average time to achieve blood glucose control was 5 hours, and elevation of serum bicarbonate was 23 hours. ${ }^{9}$ In their study, hypoglycemia was noted in $17 \%$ of patients and hypokalemia in 38\%, and average hospital stay was 13 days. ${ }^{9}$ Referral to endocrinology service was not found to improve outcome or shorten hospital stay. ${ }^{9}$

Clinical pathways or integrated clinical pathways (ICP) are evidence-based tools that serve as guides on the approach to a certain condition by detailing the steps essential to its management, ensure implementation of best practices which potentially lead to improve patient care and reduce the costs related to hospitalizations. ${ }^{6,10} \mathrm{At}$ present there is no ICP used in the management of diabetic emergencies in the Philippine General Hospital. Prior to formulating one, it would be wise to first assess the quality of care to determine if there is indeed a need for ICP.

At present, there is no scoring system or questionnaire that could be used to assess quality of care. The Donabedian's structure-process-outcome model may be used as it is believed that a good structure is observed to lead to good process which further leads to higher chances of good outcome. ${ }^{11}$ Structure is characterized by the organization itself, including material and human resources. ${ }^{11}$ Process, on the other hand, is defined as the actual practice - how care is provided by the medical staff and received by the patient. ${ }^{11}$ Finally, the effects of care define outcome. ${ }^{11}$ In 1988, Donabedian stated that before one could evaluate quality, elements that constitute it should first be defined. ${ }^{11}$ These elements include, first, the technical aspect, which is characterized by the adequacy of knowledge and judgment utilized in the planning and execution of appropriate strategies to the management of the disease and is evaluated in comparison with the accepted best practice, where best practice is defined as a strategy of care that has been known to provide the "greatest improvement in health" such that "even if the actual consequences of care in any given instance prove to be disastrous, quality must be judged as good if care, at the time it was given, conformed to the practice that could have been expected to achieve best results." 11 The second element is the interpersonal performance of the health care providers which may affect the technical aspect positively or negatively. ${ }^{11}$

\section{OBJECTIVES}

This study was conducted with the goal to evaluate the quality of care (QoC) in diabetic emergencies at the Philippine General Hospital in terms of structures, processes and outcomes, and to determine the facilitators and barriers in the effective delivery of care from the healthcare providers' standpoint.

\section{MATERIALS AND METHODS}

The first phase of this study is a retrospective chart review involving an audit of the quality of services rendered to patients diagnosed to have DKA/HHS at Philippine General Hospital, a tertiary government hospital. Review of the medical records of 70 patients diagnosed to have DKA or HHS admitted in the emergency room over a period of five years was done. Demographics such as age, sex, comorbidities, type of diabetes, severity indices, time of initiation of hydration and insulin drip, length of ICU and hospital stay, hypokalemia, hypoglycemia and mortality rates, were noted. Pregnant patients and children were excluded from this study.

The second phase of this study was characterized by a series of focus group discussions, one involving six emergency room nurses, and another with six physicians from the Departments of Emergency Medicine and Internal Medicine, and Endocrinology section of the Department of Medicine. Both discussions were conducted after informed consents were signed. These FGDs aimed to identify facilitators and barriers encountered in managing DM emergencies, and the possible reasons for such barriers. Participant responses and minutes of the meetings were recorded. 
Descriptive statistics was used to summarize the general and clinical characteristics of the participants. Frequency and proportion were used for nominal variables, median and range for ordinal variables, and mean and standard deviation for interval/ratio variables. All valid data were included in the analysis. Missing variables were neither replaced nor estimated. STATA 15.0 was used for data analysis.

\section{RESULTS}

We analyzed 70 patients with DKA/HHS. The median age was 42 (range 19-82) with majority (61\%) being females. (Table 1)

Table 2. Clinical characteristics and physical examination $(n=70)$

\begin{tabular}{|c|c|}
\hline & $\begin{array}{c}\text { Frequency (\%); Mean } \pm \text { SD; } \\
\text { Median (Range) }\end{array}$ \\
\hline $\begin{array}{l}\text { Type of diabetes } \\
\text { Type } 1 \\
\text { Type } 2 \\
\text { Unspecified }\end{array}$ & $\begin{array}{c}21(30) \\
46(65.71) \\
3(4.29) \\
\end{array}$ \\
\hline New onset diabetes & $22(31.43)$ \\
\hline $\begin{array}{l}\text { Seen by DEM/Ambu/POD within } \\
\text { an hour }[n=33]\end{array}$ & $28(84.85)$ \\
\hline $\begin{array}{l}\text { Vital signs } \\
\text { Systolic BP }[n=67] \\
\text { Diastolic BP }[n=67] \\
\text { Pulse rate, beats per min } \\
\text { Respiratory rate, cycles per min } \\
\text { Temperature, }{ }^{\circ} \mathrm{C}[\mathrm{n}=67]\end{array}$ & $\begin{array}{c}107.31 \pm 24.84 \\
68.36 \pm 14.93 \\
118.5(55-150) \\
25.5(15-56) \\
36.7(35.2-39.9) \\
\end{array}$ \\
\hline Weight $(\mathrm{kg})[\mathrm{n}=35]$ & $53.5(38-90)$ \\
\hline Height $(\mathrm{cm})[\mathrm{n}=32]$ & $157(124.46-175.26)$ \\
\hline BMI $\left(\mathrm{kg} / \mathrm{m}^{2}\right)[\mathrm{n}=30]$ & $23.11 \pm 4.52$ \\
\hline $\begin{array}{l}\text { Level of consciousness } \\
\text { Alert, awake, conscious } \\
\text { Agitated } \\
\text { Drowsy, stupurous } \\
\text { Coma }\end{array}$ & $\begin{aligned} 40 & (57.14) \\
3 & (4.29) \\
22 & (31.43) \\
5 & (7.14)\end{aligned}$ \\
\hline $\begin{array}{l}\text { Family history } \\
\text { None } \\
\text { Diabetes Mellitus } \\
\text { Hypertension } \\
\text { Bronchial asthma } \\
\end{array}$ & $\begin{array}{c}34(48.57) \\
30(42.86) \\
17(24.29) \\
1(1.43) \\
\end{array}$ \\
\hline $\begin{array}{l}\text { Past medical history } \\
\text { None } \\
\text { Diabetes Mellitus } \\
\text { Hypertension } \\
\text { PTB } \\
\text { Past DKA/HHS } \\
\end{array}$ & $\begin{array}{c}12(17.14) \\
48(68.57) \\
16(22.86) \\
11(15.71) \\
8(12.9) \\
\end{array}$ \\
\hline $\begin{array}{l}\text { Social history } \\
\text { Alcohol } \\
\text { Smoke } \\
\text { Drugs } \\
\end{array}$ & $\begin{array}{c}17(24.29) \\
12(17.14) \\
0 \\
\end{array}$ \\
\hline $\begin{array}{l}\text { Initial presenting symptom } \\
\text { Gastrointestinal } \\
\text { Neurologic } \\
\text { Respiratory } \\
\text { Cardiac } \\
\text { Others } \\
\end{array}$ & $\begin{array}{c}29(41.43) \\
19(27.14) \\
15(21.43) \\
1(1.43) \\
7(10)\end{array}$ \\
\hline
\end{tabular}

Table 1. Demographic characteristics $(n=70)$

\begin{tabular}{lc} 
& Frequency (\%); Median (Range) \\
\hline Age (years) & $42(19-82)$ \\
\hline Sex & \\
$\quad$ Male & $27(38.57)$ \\
Female & $43(61.43)$ \\
\hline
\end{tabular}

Sixty-six percent (65.71\%) of the patients had type 2 diabetes. Thirty-one percent (31\%) were newly diagnosed. Patient blood pressures were normal but pulse and respiratory rates seemed elevated. Fifty-six percent (56\%) who had a history of DM were on oral medications. Most were conscious and presented with gastrointestinal symptoms. Fifty-seven percent (57\%) were admitted in ICU. (Table 2)

\begin{tabular}{|c|c|}
\hline & $\begin{array}{c}\text { Frequency (\%); Mean } \pm \text { SD; } \\
\text { Median (Range) }\end{array}$ \\
\hline $\begin{array}{l}\text { Precipitating factor } \\
\text { None identified } \\
\text { Infection } \\
\text { Insulin related (withdrawal or } \\
\text { non-adherence) } \\
\text { Gastrointestinal } \\
\text { Others } \\
\end{array}$ & $\begin{array}{c}7(10) \\
39(55.71) \\
16(22.86) \\
10(14.29) \\
6(8.57)\end{array}$ \\
\hline Leukocytes & $20.2(2.86-101.9)$ \\
\hline $\begin{array}{l}\text { Capillary blood glucose } \\
\quad<250 \\
250-400 \\
401-600 \\
>600\end{array}$ & $\begin{array}{c}3(4.29) \\
22(31.43) \\
23(32.86) \\
22(31.43)\end{array}$ \\
\hline Random blood glucose $[\mathrm{n}=67]$ & $478.8(33.5-1349.64)$ \\
\hline $\begin{array}{l}\text { Ketones } \\
\text { Negative } \\
+1 \\
+2 \\
+3 \\
+4 \\
\text { Trace } \\
\end{array}$ & $\begin{array}{c}3(4.29) \\
4(5.71) \\
21(30) \\
31(44.29) \\
1(1.43) \\
10(14.29) \\
\end{array}$ \\
\hline Anion gap [n=62] & $27.35(10.3-57.67)$ \\
\hline Blood pH [n=69] & $7.22 \pm 14.75$ \\
\hline$B \cup N[n=69]$ & $9.4(2.2-42)$ \\
\hline $\mathrm{pCO}_{2}$ & $15.1(8.3-84.3)$ \\
\hline $\mathrm{pO}_{2}$ & $109.75(14.5-252.5)$ \\
\hline Serum bicarbonate & $6.15(3-21.5)$ \\
\hline Serum creatinine & $114.5(31-720)$ \\
\hline Serum osmolarity [n=56] & $299.35(94.5-374.54)$ \\
\hline Serum potassium & $5(1.3-8.8)$ \\
\hline Serum sodium [n=69] & $132(115-171)$ \\
\hline Central line insertion & $10(14.29)$ \\
\hline $\begin{array}{l}\text { Admission } \\
\text { ER } \\
\text { ICU } \\
\text { Ward } \\
\end{array}$ & $\begin{array}{c}6(8.57) \\
40(57.14) \\
24(34.29) \\
\end{array}$ \\
\hline Admitted within 24 hours [ $n=65]$ & $46(70.77)$ \\
\hline $\begin{array}{l}\text { Severity of DM }[\mathrm{n}=61] \\
\text { HHS } \\
\text { Mild } \\
\text { Moderate } \\
\text { Severe }\end{array}$ & $\begin{array}{c}1(1.64) \\
7(11.48) \\
14(22.95) \\
39(63.93) \\
\end{array}$ \\
\hline
\end{tabular}


The median hospital stay of patients with DKA was one week (range 1 to 58 days) and the median ICU stay was three days (range 1 to 22 days). Of the seventy (70) patients, sixteen (16) died. (Table 3)

Table 3. Clinical course of DKA/HHS patients $(n=70)$ Median (Range); Frequency (\%)

\begin{tabular}{lc} 
Length of hospital stay, days & $7(1-58)$ \\
\hline Length of ICU stay, days $[\mathrm{n}=44]$ & $3(1-22)$ \\
\hline Outcome & \\
Discharged & $51(72.86)$ \\
HAMA & $3(4.29)$ \\
Mortality & $16(22.86)$ \\
\hline
\end{tabular}

Eighty-four percent (83.82\%) followed the prescribed guidelines on the volume of fluid infusion during the first 24 hours. Almost half (48.57\%) of the patients had no incidence of hypoglycemia while $20 \%$ did not have hypokalemia episode. (Table 4)

Table 4. Quality Indicators: Management of Diabetic Emergencies and comparison to recommended guidelines

\begin{tabular}{lc} 
& $\begin{array}{c}\text { \% of cases following } \\
\text { guidelines }\end{array}$ \\
$\begin{array}{l}\text { Hydration } \\
\text { Initiation within } 30 \text { minutes [n=45] } \\
\text { Correction of half the water deficit } \\
\text { (at least } 3 \text { Liters of fluid) within the first }\end{array}$ & $19(42.22)$ \\
$\quad 24$ hours [n=68] & \\
\hline $\begin{array}{l}\text { Insulin drip initiated after hydration has been } \\
\text { done [n=40] }\end{array}$ & $39(97.50)$ \\
\hline $\begin{array}{l}\text { Biochemical testing done at least 6x in the } \\
\text { first 24 hours of admission [n=37] }\end{array}$ & $7(10)$ \\
\hline No Hypoglycemia episodes & $34(48.57)$ \\
\hline No Hypokalemia episodes & $14(20)$ \\
\hline Referral to Endocrine Service & $68(97.14)$ \\
\hline
\end{tabular}

Forty-seven (47), or $67 \%$ of the seventy patients were considered to have DM emergency on first encounter at the emergency room. Twenty-nine percent (29\%) died among the patients who were seen within an hour of admission, while $60 \%$ mortality rate was observed among patients seen more than an hour after arrival at the ER. In patients with delayed initiation of hydration (more than 30 minutes from admission), 23\% mortality rate was observed; and as much as $50 \%$ and $77 \%$ of these patients had at least one episode of hypoglycemia or hypokalemia, respectively. It was also noted that failure of documenting the time of initiation of hydration was observed in $36 \%$ of cases. Only $10 \%$ had monitoring of electrolytes at least six (6) times within the first twenty-four (24) hours of admission. In $97 \%$ of cases, insulin drip was started after initiation of hydration. Over-all mortality was 23\%. Among those who died, seventy-five percent (75\%) and over thirty percent (31\%) had at least one episode of hypokalemia or hypoglycemia, respectively.

\section{Focus Group Discussion}

In the first FGD, the physicians mentioned that recognizing the disease had not been a problem and they were all aware of the guidelines. However, challenges were experienced in the delivery of care itself. The physicians followed the treatment algorithm for DM emergencies by Kitabchi and Umpierrez which they have saved in their smart phones. The study participants have identified the lack of resources such as capillary blood glucose (CBG) testing strips, certain intravenous fluids (IVF) and limited bed capacity compared to the number of patients requiring admission as common challenges in DM emergency management. The average time laboratory results become available was more than 4 hours, which prompted the clinicians to request tests every 4 to 6 hours instead of the recommended 2 to 4 hours. The delays were attributed to the handling of the specimens where a clerk or aide personally delivered the samples in batches from the ER to the laboratory department. When the doctors were asked regarding measures that may be employed to improve the quality of care, all of the participants positively responded for the development of a pathway that is concise, easy to follow and one that "won't be an added paperwork." Provision of a DM emergency monitoring sheet (a combined vital signs and fluid monitoring sheet, and laboratory flow chart) and a print out of the treatment algorithm may be sufficient according to them. The physicians also mentioned that a "DM emergency stamp" may be used to label the requests for serial laboratory examinations and pharmacy requests to "alert" clerks, technicians, pharmacists and all members of the team to facilitate the tests without delay. (Table 5)

\section{Table 5. Summary of FGD findings}

Physicians did not have difficulty recognizing a DM emergency as they were given lectures on DM emergency management as part of their training

Nurses admitted to having limited knowledge on the manifestations and management of DKA/HHS

Delays in the management of DKA/HHS were attributed to:

- Lack of resources (specifically glucometers and strips, IV fluids)

- Limited bed capacity (ICU beds)

- Handling of specimens from the ER clerk to the laboratory (total time 4-6 hours to deliver specimen and retrieve results)

Recommendations to improve quality of care

- Provision of "DM Emergency Kits" for each patient

- DM Emergency monitoring forms

- DM Emergency stamp/alert/clinical pathway

- Education and training for nurses regarding the manifestations and management of DM emergencies

The second FGD was attended by the emergency medicine nurses. It was noted that their knowledge on the disease and management was limited, specifying blood sugar elevation and decrease in sensorium as the only recognizable presentation that they were aware of. All of the participants 
mentioned that they do not have trainings and lectures on the subject and that they have learned the management of the disease only by observing the orders made by different physicians. It was mentioned that they were able to carry out orders of the physicians without delay despite each nurse handling $15-20$ patients in one shift. Their limitation was on the unavailability of some resources such as $\mathrm{CBG}$ testing strips, certain IVF $(0.45 \mathrm{NaCl})$ and limited number of glucometers, where only two were shared in the ER, one for adults and another for pediatric patients. When asked about actions or changes that could possibly improve the quality of care for DM emergency management, the nurses proposed a "DM emergency kit" which will contain a glucometer, CBG testing strips, lancets, insulin, IVF fluids (PNSS, PLR, and $0.45 \mathrm{NaCl}$ ), IV set, and monitoring sheets. (Table 5)

\section{DISCUSSION}

DKA and HHS are life-threatening acute complications of both type 1 and type 2 diabetes mellitus with varying presentations and precipitating factors. ${ }^{1,5}$ DKA is characterized by elevation of blood sugar, acidosis and positive ketones while the diagnosis of HHS is made when elevated blood sugar and effective osmolarity, normal $\mathrm{pH}$ and decreased sensorium are present. ${ }^{1,3,4}$ The most common precipitating factor in the study by Efstathiou, et al. in Greece is insulin withdrawal which is different from that in the US, and in Indonesia where infection was reported in as much as $80-90 \%$ of cases. ${ }^{4,12,13}$ Similarly in our study, infection was also the main precipitating factor observed in $56 \%$ of cases, insulin withdrawal or insulin non-adherence comes second at $23 \%$. This was consistent with the findings of Gatbonton et al. in 1998, where infection was a precipitating factor observed in $38 \%$ of the sample..$^{9}$ Identification and management of these precipitating factors are important in the treatment of DM emergencies. The guidelines recommend that at the $24^{\text {th }}$ hour admission these precipitating factors should have already been addressed to improve the outcomes. ${ }^{3}$

DKA mortality rates are as low as $<1 \%$ to $3.3 \%$ in the UK, US and Canada, but as much as 30\% in "less privileged health systems." ${ }^{4-8}$ This may be even higher in HHS cases. ${ }^{1}$ A study in Indonesia reported a seventy-two (72) hour mortality rate as high as $29 \% .{ }^{13}$ On the other hand, an eleven percent rate (11\%) was reported in the Philippines in 1998 which was half the rate that was observed in this study which was $23 \%{ }^{9}$

Effective management are possible through adequate fluid resuscitation, insulin therapy, correction of electrolyte imbalances and close monitoring of the patient with use of laboratory procedures to assess resolution. ${ }^{1}$ Water deficit in DKA patients is approximately six (6) liters, hence the goal of initial fluid therapy is to replace at least half of the estimated water deficit, at least three liters (3L), within the first 12 to 24 hours of admission to expand intravascular volume while ensuring adequate urine output. ${ }^{1,3}$ The fluid of choice depends on corrected sodium and glucose where $0.45 \% \mathrm{NaCl}$ is preferred in patients with normal or high sodium, and $0.90 \% \mathrm{NaCl}$ in cases of hyponatremia. ${ }^{1}$ Once sugars reach $250 \mathrm{mg} / \mathrm{dL}$ in DKA, fluids should be shifted to glucose containing half saline to prevent rapidly correcting hyperglycemia as this could lead to cerebral edema. ${ }^{1}$ After adequate hydration is given, insulin therapy is initiated with IV bolus of regular insulin at $0.1 \mathrm{u} / \mathrm{kg}$, followed with $0.1 \mathrm{U} /$ $\mathrm{kg}$ as continuous drip every hour, and is withheld if serum $\mathrm{K}$ is $3.3 \mathrm{mmol} / \mathrm{L}$ and below. ${ }^{1}$ Bicarbonate therapy is not recommended unless $\mathrm{pH}$ is less than 7.0. ${ }^{1}$ Blood draws is recommended every 2-4 hours to check for serum electrolytes, blood urea nitrogen, creatinine, serum osmolarity and venous $\mathrm{pH} .{ }^{1}$ When the blood glucose is $<200 \mathrm{mg} / \mathrm{dl}$, serum bicarbonate is 15 and above, $\mathrm{pH}$ is $>7.30$ and anion gap is 12 or less, DKA is resolved. ${ }^{1}$ It is also recommended that by the $24^{\text {th }}$ hour of hospitalization, a referral to a diabetes educator should be made as this is vital in decreasing the chances of readmission. ${ }^{3}$ Although these recommendations have already been made to guide healthcare providers in delivering care for DM emergency patients, it has been previously reported that these guidelines were followed only $22 \%$ of the time which could lead to poorer outcomes. ${ }^{5}$

Since there is no available scoring system yet to assess the quality of care, Donabedian proposes the use of the structure-process-outcome model. ${ }^{11}$ This looks into the adequacy of knowledge and judgment utilized in the planning and execution of appropriated strategies to manage the disease. ${ }^{11}$ It also evaluates and compares the system's structures, processes and outcomes to be accepted best practices. ${ }^{11}$ Donabedian further states that "even if the actual consequences of care in any given instance prove to be disastrous, quality must be judged as good if care, at the time it was given, conforms to the practice that could have been expected to achieve best results." ${ }^{11}$

In this study it was found that in the Philippine General Hospital, initiation of hydration was done within 30 minutes of admission in $40 \%$ of cases. Only $10 \%$ of patients had adequate laboratory monitoring, and correction of at least half of the estimated water deficit was achieved in $84 \%$ of cases. Despite achieving correction of at least half of the water deficit within the prescribed time in most cases, mortality rate was still very high at $23 \%$. The discordance between these results may be attributed to the finding that consideration for the diagnosis of a diabetic emergency at first encounter with the healthcare physician was only observed in $67 \%$ of cases and that timely initiation of hydration was observed in less than half of the cases. The nurses also admitted to lack knowledge in the presentation and management of DKA/ HHS. FGD also revealed that delays were experienced in transferring patients from the triage area to the ER consultation area due to the lack of beds and manpower. Although these delays cannot be accurately evaluated in the study due to missing data because most of the healthcare 
providers in-charge failed to put time stamps on their order sheets, these could still have contributed to the seemingly higher mortality rates observed. Poor documentation could have also contributed to poor delivery of quality care because a lot may be missed, which may be detrimental to the patient. Delays in the laboratory procedures and releases of the results were also mentioned in the discussion which led the clinicians requesting laboratory tests every 4-6 hours instead of the recommended 2-4 hours. Literature states that death in DKA most likely occurred in the first 72 hours of admission, hence the timing is important. ${ }^{12}$ Delays must be avoided. Timely and efficient delivery of treatment must be observed. The key to successful resolution of these emergencies include early recognition and diagnosis, comprehensive evaluation, both clinical and biochemical, and effective management. ${ }^{1}$

Recommendations were formulated to address the identified barriers to improve the quality of care. The participants of the FGDs have suggested formulation of a pathway, provision of DM emergency kits, and utilization of DM emergency alerts/stamps, and improvement in the education of healthcare providers to address these barriers. Integrated Care Pathways or ICPs are "management plans that display goals for patients and indicate the sequences and the timing of actions" needed to manage a certain condition. These pathways have the potential to improve patient care and reduce costs related to hospitalizations. ${ }^{6,10}$ In a 2007 study by Waller et al, a clinical pathway for the treatment of DKA was implemented in a teaching hospital, and this led to shorter time from triage to initiation of fluids and insulin. ${ }^{10}$ However the length of hospital stay and rates of hypokalemia or hypoglycemia and hospital costs did not significantly differ. ${ }^{10}$ They still proposed that avoidance of delays could lead to lower rates of complications and faster recovery time. ${ }^{10}$ Having a clinical pathway tailor-fit to the PGH setting may be utilized to improve our delivery of care to our patients.

\section{CONCLUSION}

Failure to follow guidelines and delays in the delivery of care are possible reasons for the high mortality rates noted and could be a reflection of poor quality of care among DM emergency patients in PGH. Proper documentation in the medical charts is also important. Clinical pathways and DM emergency kits are a few of the suggested approach to address the barriers to good quality care.

\section{Limitations and recommendations}

One of the challenges encountered in the conduct of this study was the difficulty in retrieving the charts, their completeness and the quality of the records, e.g. most of the charts reviewed had missing time stamps, hence, the resultant small sample size.

\section{Statement of Authorship}

All authors participated in the data collection and analysis, and approved the final version submitted.

\section{Author Disclosure}

All authors declared no conflicts of interest.

\section{Funding Source}

This paper was funded by the Philippine General Hospital Research Grant 2018.

\section{REFERENCES}

1. Gosmanov AR, Gosmanova EO, Kitabchi AE. Hyperglycemic Crises: Diabetic Ketoacidosis (DKA), and Hyperglycemic Hyperosmolar State (HHS). In: Feingold K, Anawalt B, Boyce A, Al E, eds. Endotext [Internet]. 2018 May [cited 2017 Oct]. Available from: https://www. ncbi.nlm.nih.gov/books/NBK279052/?report=printable.

2. American Diabetes Association. Diabetes care in the hospital: standards of medical care in diabetes-2019. Diabetes Care. 2019; 42(Suppl 1):S173-S181. doi:10.2337/dc19-S015

3. Gosmanov AR, Gosmanova EO, Dillard-Cannon E. Management of adult diabetic ketoacidosis. Diabetes Metab Syndr Obes. 2014;7: 255-64. doi:10.2147/DMSO.S50516

4. Kitabchi AE, Umpierrez GE, Miles JM, Fisher JN. Hyperglycemic crises in adult patients with diabetes. Diabetes Care. 2009; 32(7): 1335-43. doi:10.2337/dc09-9032

5. Singh RK, Perros P, Frier BM. Hospital management of diabetic ketoacidosis: are clinical guidelines implemented effectively? Diabet Med. 1997;14(6):482-6. doi:10.1002/(SICI)1096-9136(199706)14: 6<482::AID-DIA371>3.0.CO;2-A

6. Hassan IS, Al-Otaibi AD, Al-Bugami MM, Salih SB, Saleh YA, Abdulaziz S. The impact of a structured clinical pathway on the application of management standards in patients with diabetic ketoacidosis and its acceptability by medical residents. J Diabetes Mellitus. 2014; 4(4):264-72. doi:10.4236/jdm.2014.44038

7. Gibb FW, Teoh WL, Graham J, Lockman KA. Risk of death following admission to a UK hospital with diabetic ketoacidosis. Diabetologia. 2016; 59(10):2082-7. doi:10.1007/s00125-016-4034-0

8. Goguen J, Gilbert J. Hyperglycemic emergencies in adults. Can J Diabetes. 2013; 37 Suppl 1:S72-6. doi:10.1016/j.jcjd.2013.01.023

9. Gatbonton PB, Lim-Abrahan MA. Diabetic ketoacidosis: a five year review at the Philippine General Hospital. Philipp J Intern Med. 1998; 36(2):13-7.

10. Waller SL, Delaney S, Strachan MWJ. Does an integrated care pathway enhance the management of diabetic ketoacidosis? Diabet Med. 2007; 24(4):359-63. doi:10.1111/j.1464-5491.2007.02102.x

11. Donabedian A. The quality of care: how can it be assessed? JAMA. 1988; 260(12):1743-8. doi:10.1001/jama.1988.03410120089033

12. Efstathiou SP, Tsiakou AG, Tsioulos DI, Zacharos ID, Mitromaras AG, Mastorantonakis SE, et al. A mortality prediction model in diabetic ketoacidosis. Clin Endocrinol (Oxf). 2002; 57(5):595-601. doi:10.1046/j.1365-2265.2002.01636.x

13. Siregar N, Soewondo P, Subekti I, Muhadi M. Seventy-two hour mortality prediction model in patients with diabetic ketoacidosis: A retrospective cohort study. J ASEAN Fed Endocr Soc. 2018; 33(2):124-9. doi:10.15605/jafes.033.02.03 